\title{
Impurity Profiling of Pharmaceutical Drugs By Various Methods
}

\author{
Shwetali K. Churi ${ }^{1}{ }^{2}$ Manohar V. Lokhande ${ }^{3 *}$ \\ ${ }^{1}$ Research Scholar, Shri Jagdishprasad Jhabarmal Tibrewala University, Jhunjhunu-333001, Rajasthan, India. \\ ${ }^{2}$ Department Of Chemistry, Viva Institute Of Technology, Shirgaon, Virar(East),Vasai, Dist-Palghar-401305, \\ Maharashtra, India. \\ ${ }^{3 *}$ Department Of Chemistry, Sathaye College, Vile Parle (E), Mumbai-400057, Maharashtra, India
}

\begin{abstract}
Analysis is the basic in every product or service and is very essential in drugs as it deals with life. Analytical chemistry is the study of material with respect to its qualitative and quantitative composition. In order to ensure the safety and efficacy of all drugs throughout all phases of its life shelf, considering its storage, distribution and use, every pharmaceutical product should be analytically monitored to find the impurity level in any pharmaceutical drug with the help of new techniques and methodologies like UV Spectroscopy, IR Spectroscopy, Mass Spectrometry, NMR spectroscopy and HPLC Methods.
\end{abstract}

Keywords: Validation of Drug, HR/AM/MS , LOD, LOQ,LC-MS and HPLC

\section{Introduction}

Impurity profiling or Characterization refers to the broad and general process to refer any materials analysis process including macroscopic techniques such as mechanical testing, thermal analysis and density calculation which ranges from angstroms to centimeters, such as in the imaging of coarse grain structures in metals and finding the impurity level in any pharmaceutical drug with the help of new techniques and methodologies. a) Ultraviolet (UV)Spectroscopy, b) Infrared (IR)Spectroscopy, c) Mass Spectrometry (MS), d) Nuclear Magnetic Resonance (NMR), e) HPLC Methods[1-2]. Various regulatory authorities like United States Food Corporation and Drug Authority (USFDA), European Directorate of Quality Medicine (EDQM), Therapeutic Goods administration' World Health Organization (WHO) and other health agencies are emphasizing on the purity requirements. A key component of the overall quality of a pharmaceutical is control of impurities, as their presence, even in small amounts, might also affect safety and efficacy of drug. International Conference on Harmonization of Technical Requirements for Registration of Pharmaceuticals for Human Use (ICH) guidelines are developed [3] with the joint efforts of regulators and industrial representatives from the European Union, Japan and the United States. The guidelines helped to ensure that, the unlike regions have consistent necessities for the record that should be submitted to the various regulatory agencies. As per ICH Q3A(R) and ICH Q3B $(R)$ guidelines, unknown impurities related to bulk drug and dosage form, more than the identification threshold must be identified[4].Sources of Impurities: The different sources of impurities are given below:

Crystallization-related impurities: Residual solvents or organic volatile chemicals occur during crystallization of the bulk drug substance.

Impurities originating from synthetic processes of the drug substance: Starting materials and intermediates are the chemical constructing blocks used to construct the final form of a drug substance molecule. Un-reacted starting materials and intermediates can potentially survive the purification process and appear in the final product as impurities, by-products formed from the side-reactions in drug synthesis accounts the common impurities in drugs. Chemically active reagents, metallic ligands, and various catalysts used in the synthesis of a drug substance can be carried over to the final products as trace level impurities.

Impurities arising during storage: Degradation of the drug substance is one of the main sources of impurities caused by chemical instability of the drug substance under the conditions (e.g., heat, moisture, solvent use, $\mathrm{pH}$, light, etc.) of manufacturing, isolation, purification, drying, storage, transportation, and interactions with other chemical entities in the formulation.

Identification and Characterization of Impurities: Initial step is to identify the impurities by different methods like reference standard method, separation methods and isolation method, further the characterization of impurities can be carried out using spectroscopic, spectrometric and hyphenated techniques.

Reference standard method: If the initial analysis shows that the observed impurity falls into the standard impurity category, the impurity identification turns into practice which can be achieved by three experiments analysis of sample, followed by analysis of standard and lastly mixture of sample with standard by applying any of chromatographic or spectroscopic techniques.

Separation methods: The methods used for the separation of impurities and degradation products are generally as follows high performance liquid chromatography (HPLC), gas chromatography (GC) etc.

\begin{tabular}{lll}
\hline DOI: $10.9790 / 5736-1007012734$ & www.iosrjournals.org & 27 |Page
\end{tabular}


High Performance Liquid Chromatography (HPLC): This Chromatography technique is a multistage separation method where the components are divided in two phase's i.e. stationary phase (solid in nature and packed in column) and mobile phase (liquid in nature and freely flowing). Individual component in the injected drug has different response (to both the phases) and different rate of migration while passing through column and resulting in different partition. Compound having less affinity elutes first in comparison to the compound having better affinity with recognize to stationary phase [5].

Gas chromatography (GC): GC is a dynamic method used for separation and detection of volatile organic compounds in a mixture. It involves the phasing of gaseous solutes between an inert gas mobile phase and stationary solid or liquid phase. A large number of volatile components can be separated by modern capillarycolumn gas chromatographs, which permits identification through retention characteristics and detection at ppm levels using a wide range of detectors [6].

Isolation methods: It is necessary to separate impurities for their structural elucidation. Generally chromatographic and non-chromatographic techniques are used for isolation of impurities before characterization. A list of methods that can be used for isolation of impurities is given below.

(i) Solid phase extraction method Liquid-liquid extraction method (ii) solvent extraction method (iii) Supercritical fluid extraction (iv) Column chromatography (v) Preparative HPLC (vi) Preparative TLC(vii) Flash chromatography

Characterization methods (Spectroscopic, Spectrometric and Hyphenated Techniques): Ultraviolet (UV), infrared (IR), mass spectrometry (MS) and nuclear magnetic resonance (NMR) spectroscopic methods are widely used methods for the characterization of synthesized or isolated impurities. It is been observed that various functional groups give characteristic IR absorption at specific ranges, in complex atomic interaction within the molecule, IR absorption of the functional groups may vary over a wide range. IR spectral data is a confirmatory test for information obtained from other techniques.

Nuclear magnetic resonance: NMR plays a vital role in identifying low level impurities in bulk drug materials with or without chromatographic isolation. Structural elucidation of impurities in drug materials mostly involve ${ }^{1} \mathrm{H}$ and ${ }^{13} \mathrm{C}$ experiments, the information obtained from these experiments is sufficient to ascertain the structure of the unknown impurity in the drug material. The introduction of NMR with on-line coupling to HPLC reduces the need for preparative isolation of impurities. HPLC analysis is carried out in the reverse - phase mode using $\mathrm{D}_{2} \mathrm{O} /$ buffer - acetonitrile based eluents with a higher injection volume (50-100ul)[7].

Mass Spectrometry in Impurity Profiling: The technique where charged species (ions) can be separated and detected according to their mass to charge ratio $(\mathrm{m} / \mathrm{z})$ is known as Mass spectrometry (MS). MS is one of the highly selective and most sensitive methods for molecular analysis giving insights to the structure of the analyte. Coupling of separation techniques, like chromatography, to MS further increases the power of the technique when complex samples are to be analyze[8].

Accuracy Study: The true value for accuracy assessment can be obtained in several ways. One alternative is to compare the results of the method with results from an established reference method. This approach assumes that the uncertainty of the reference method is known. Secondly, accuracy can be assessed by analyzing a sample with known concentrations (e.g., a control sample or certified reference material) and comparing the measured value with the true value as supplied with the material. If certified reference materials or control samples are not available, a blank sample matrix of interest can be spiked with a known concentration by weight or volume. After extraction of the analyte from the matrix and injection into the analytical instrument, its recovery can be determined by comparing the response of the extract with the response of the reference material dissolved in a pure solvent. Because this accuracy assessment measures the effectiveness of sample preparation, care should be taken to mimic the actual sample

Preparation as closely as possible. If validated correctly, the recovery factor determined for different concentrations can be used to correct the final results.

Precision Study: "The Precision of an analytical method is the degree of repeatability of the results in a series of experiments run during a single session by a single operator with identical reagents and equipment". The Precision study is carried out by calculating the \% RSD of peak area of standard solution and Test solution.

Specificity Study:"The Specificity of an analytical method is its ability to measure accurately and specifically the analyte in the presence of component that may be expected to be present in the sample matrix. Specificity is a measure of the degree of interference (or absences thereof) in the analysis of complex sample mixture."

Limit of Detection: The limit of detection is the point at which a measured value is larger than the uncertainty associated with it. It is the lowest concentration of analyte in a sample that can be detected but not necessarily quantified. The detection limit is the injected amount of sample that results in a peak with a height at least two or three times as high as the baseline noise level.

Limit of Quantitation: The limit of quantitation is the minimum injected amount that produces quantitative measurements in the target matrix with acceptable precision in chromatography, typically requiring peak heights 10 to 20 times higher than the baseline noise. A number of samples with decreasing amounts of the analyte are 
injected six times. The calculated RSD percent of the precision is plotted against the analyte amount. The amount that corresponds to the previously defined required precision is equal to the limit of quantitation.

Linearity and Range Study: The linearity of an analytical method is its ability to elicit test results that are directly proportional to the concentration of analytes in samples within a given range or proportional by means of well-defined mathematical transformations. Linearity is determined by a series of 3 to 6 injections of 5 or more standards whose concentrations span 80-120 percent of the expected concentration range.

Range: The range of an analytical method is the interval between the upper and lower levels (including these levels) that have been demonstrated to be determined with precision, accuracy and linearity using the method as written [9-10].

Ruggedness Study: Ruggedness is a measure of reproducibility of test results under normal, expected operational conditions from laboratory to laboratory and from analyst to analyst. Ruggedness is determined by the analysis of aliquots from homogeneous lots in different laboratories. The ruggedness of the method was carried out by two different analysts on two different instruments in two different labs on different dates to know the degree of reproducibility of the results obtained by the analysis of the same samples as well as system suitability parameters.

Robustness: "The robustness of an analytical method is measure of its capacity to remain unaffected by small but deliberate variations in method parameters and provides an indication of its reliability during normal usage" Robustness tests examine the effect that operational parameters have on the analysis results. For the determination of a method's robustness, a number of method parameters, for example, $\mathrm{pH}$, flow rate, column temperature, injection volume, detection wavelength or mobile phase composition, are varied within a realistic range, and the quantitative influence of the variables is determined. If the influence of the parameter is within a previously specified tolerance, the parameter is said to be within the method's robustness range.

\section{Experimental}

Chemicals and reagents: The samples of Dimer Impurity were collected from Pharmaceutical Company, Mumbai, and Maharashtra, India. GCMS grade Dimethyl Sulfoxide was purchased from Advent, Mumbai, India.

Equipment: The GCMS method development and validation were done using Thermo Scientific Trace 1310 and TSQ 8000 Connected with Thermo Scientific mass detector GCMS system. The data were collected using Chromeleon ${ }^{\mathrm{TM}}$ 6.8 Chromatography Data System (CDS) Software.

\section{Result And Discussion}

Identification of impurities by LC/HR/AM/MS: LC/HR/AM/MS and MSMS were performed as per the method described in Liquid chromatography-high resolution accurate mass spectroscopy (LC/HR/AM/MS) to generate the mass data for the impurity [11]. The impurity of interest exhibits a protonated molecular ion peak $[\mathrm{M}+\mathrm{H}]{ }^{+}$385.05156(Fig 1). The theoretical atomic formula probability shown by Xcalibur software for 385.05106 was $\mathrm{C}_{20} \mathrm{H}_{14} \mathrm{Cl}_{2} \mathrm{~N}_{2} \mathrm{O}_{2}$ PPM error was calculated using formula:

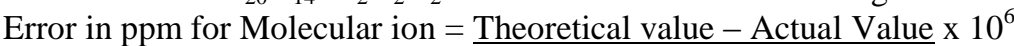

$$
\begin{gathered}
\text { Theoretical Value } \\
=\frac{385.05106-385.05156}{385.05106} \times 10^{6} \\
=1.298 \mathrm{ppm}
\end{gathered}
$$

$1.298 \mathrm{ppm}$ error is highly acceptable for characterization of $[\mathrm{M}+\mathrm{H}]^{+}$of unknown structure using HR/AM/MS. Error in ppm for MS/MS (Daughter ion) $=$ Theoretical value - Actual Value $\times 10^{6}$

$$
\begin{aligned}
& \quad \begin{array}{l}
\text { Theoretical Value } \\
=\frac{351.08948-351.08862}{351.08948} \\
=2.449 \mathrm{ppm}
\end{array}
\end{aligned}
$$

2.449ppm error is highly acceptable for characterization of MS/MS of unknown structure using HR/AM/MS.

Though the mass error was low it was mandatory to confirm the structure by NMR which would provide us the information of exact proton and carbon. Hence NMR of impurity and the product was carried for further confirmation of structure and the product was carried for further confirmation of structure.

Analytical Method Validation by HPLC: The validation study allowed the evaluation of the method for its suitability for regular analysis. The newly developed method for Dimer impurity was validated according to ICH guidelines. A typical chromatogram showing Dimer impurity (Fig.3).

Specificity: Specificity is the ability of analytical method to measure the analyte response in the presence of its potential impurities and degradants. The specificity of the Acquity H-class liquid method was determined by injecting Dimer impurity, wherein no interference was observed for any of the components from sample [1213]. 
Precision: The precision of the method was examined using six replicate injections of a standard solution. The relative standard deviation (RSD) was calculated for response (area) of Dimer impurity. The RSD for Dimer impurity was found to be $0.97 \%$ refer (Table 2) and the method precision was established by analyzing using six different preparations [14]. The calculated RSD of these results was found to be within acceptable limit (Fig.4) . Accuracy: The accuracy of the method was determined for the related substances by spiking of known amounts of Dimer impurity at levels, LOQ, $80 \%, 100 \%$ and $120 \%$ of the specified limit. The recoveries of impurities were calculated and are given in (Table 2) and (Fig.5).

Limit of detection and limit of quantification: Detection limit (DL) and quantitation limit (QL) was estimated as per ICH Q2RI. The limit of detection established for Dimer impurity was found to be 0.0197ppm i.e. $0.012 \%$ and limit of quantification was found to be $0.0598 \mathrm{ppm}$ i.e. $0.004 \%$ with respect to test solution 500ppm ( Table 3 and Fig.6)

Linearity: Linear calibration curve were obtained over the calibration range i.e. LOQ, 50\%, 80\%, $100 \%, 120 \%$ and $150 \%$ at six concentration levels in triplicate. The results showed excellent correlation between the peak area and concentration of Dimer impurity of about 0.9990 ( Table 4 and Fig.7).

Robustness: In all the deliberately varied chromatographic conditions (column temperature $\pm 3^{\circ} \mathrm{C}$ and flow rate $\pm 0.1 \mathrm{ml} / \mathrm{min}$ ), no significant changes in results were observed.

Solution stability: The solution stability of sample and Dimer impurity was carried out by keeping both solutions in tightly capped HPLC vials at $25^{\circ} \mathrm{C}$ for 48 hours in an Acquity H-class auto sampler no significant changes were observed in the peak area up to 23 hours. Successfully validated for control of 1, 1-bis-(7-chloro-4hydroxy-3-quinolyl -ethane in API.

IV. Tables And Figures

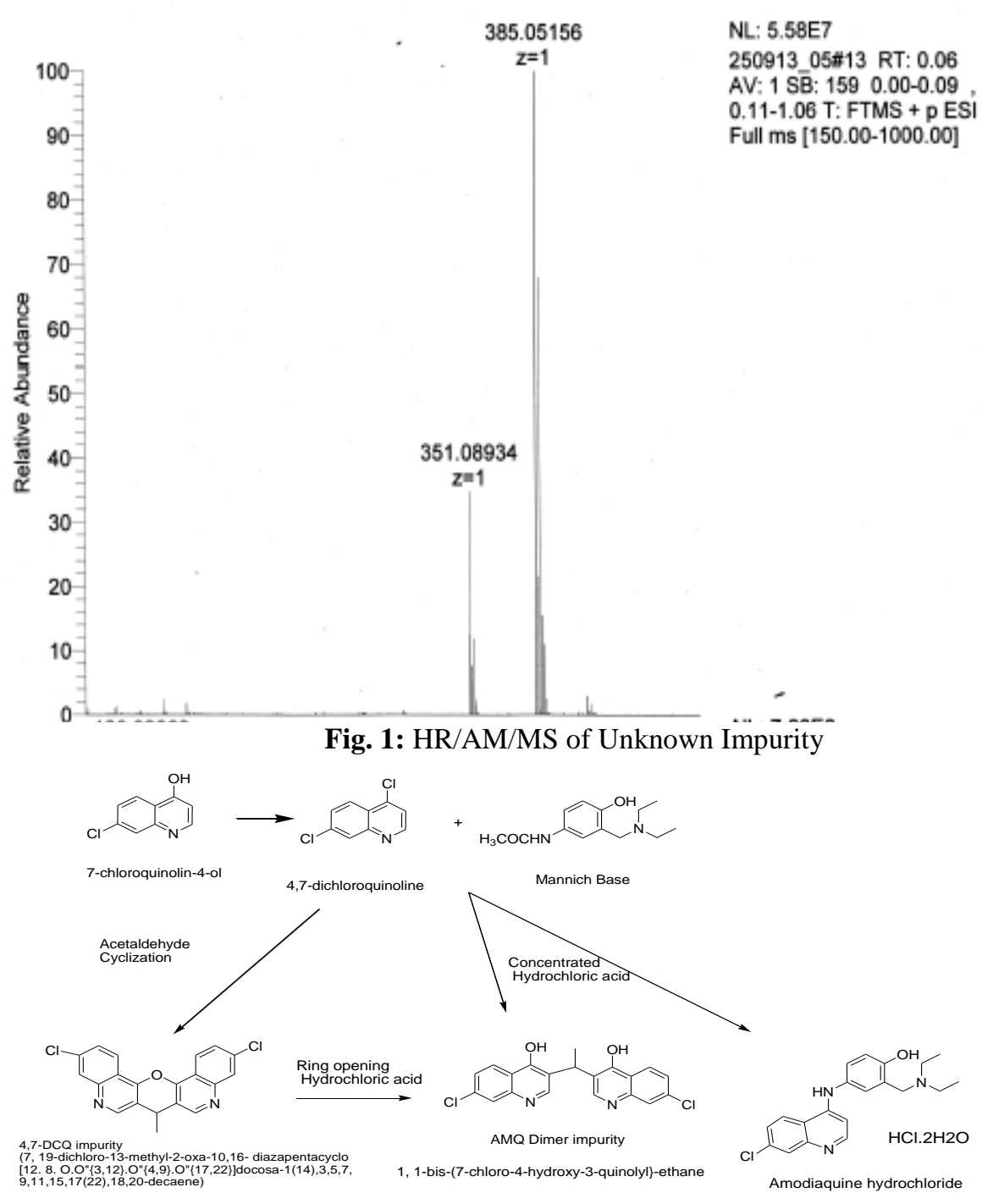

Fig.2: Scheme For Formation of Impurity1, 1-Bis-(7-Chloro-4-Hydroxy-3-Quinolyl\}-Ethane 


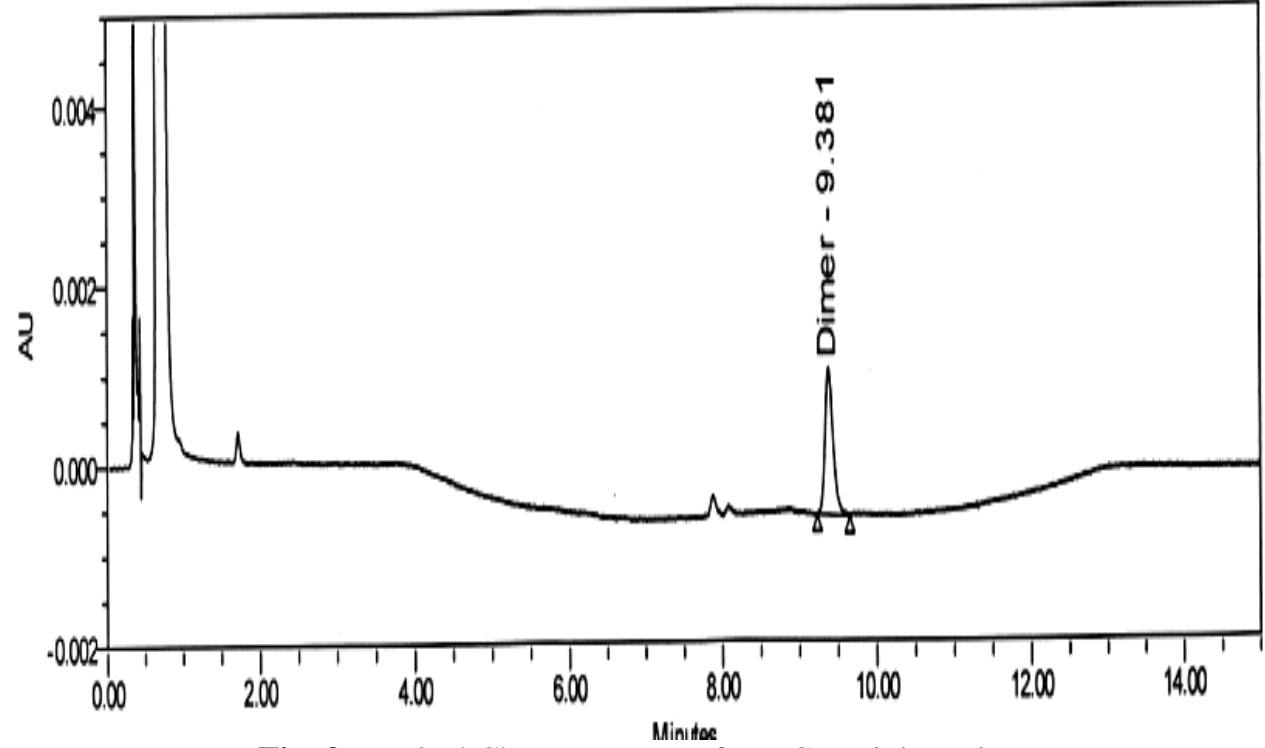

Fig. 3: Typical Chromatogram of test Containing Dimer

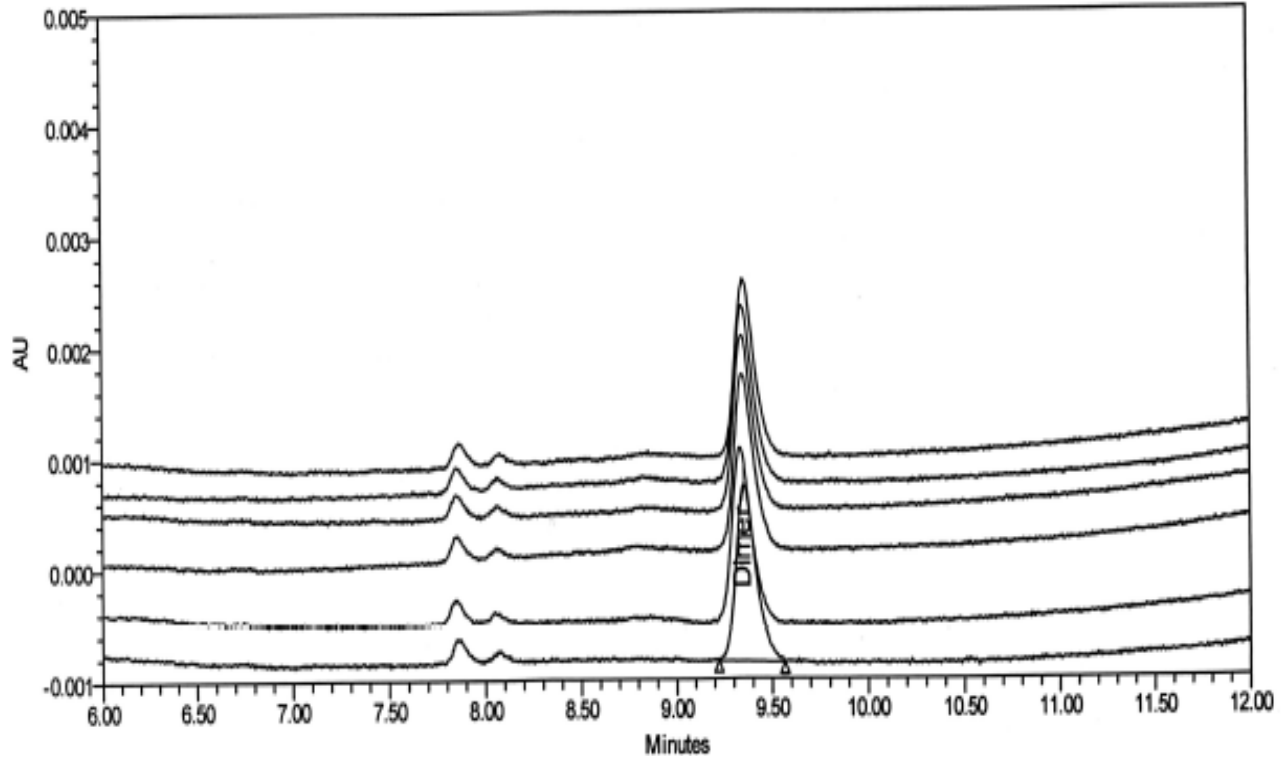

Fig. 4: A Typical Overlay Chromatogram of Six Replicate of Impurity for Precision Study

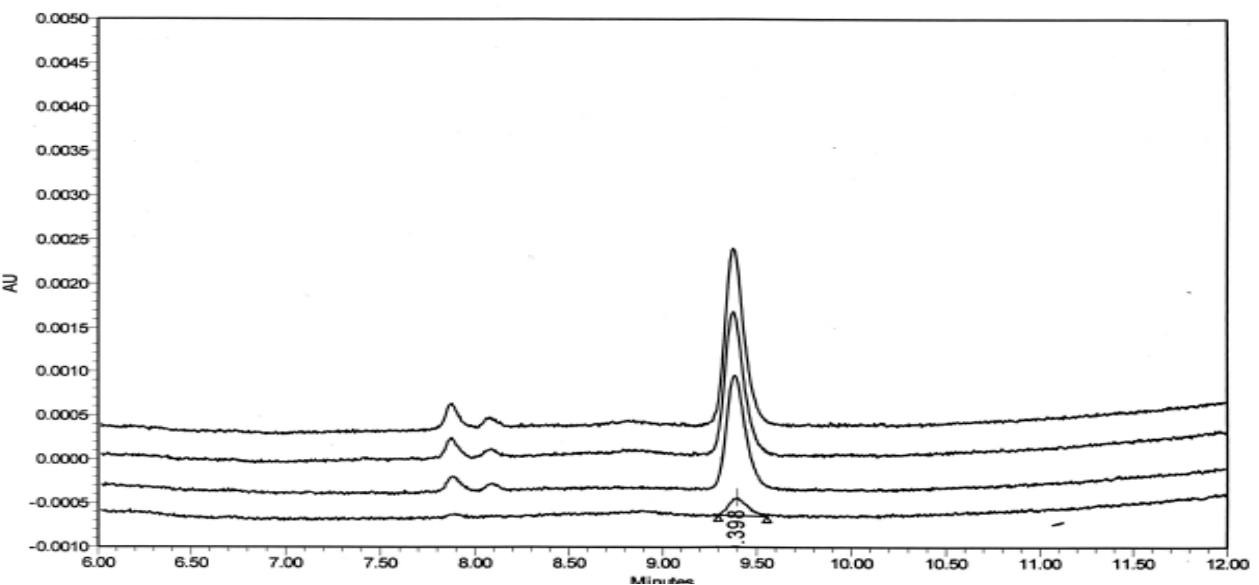

Fig .5: Typical Overlay Chromatogram of Impurity for Accuracy Study at Level LOQ, 80\%, 100\% \& 120\% 


\section{LOD AND LOQ OF DIMER}

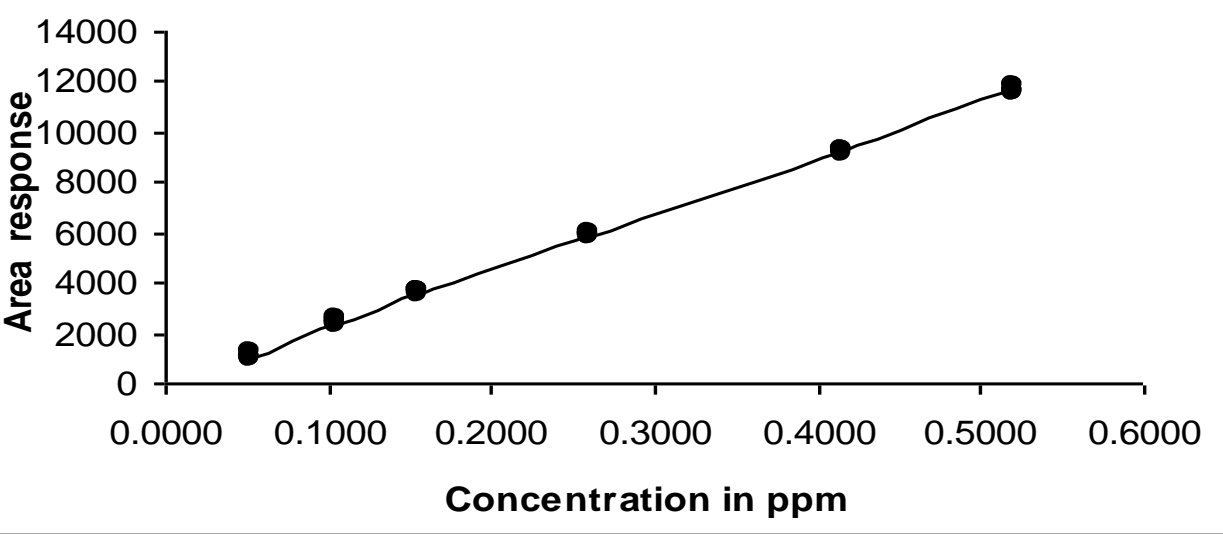

Fig.6: Linearity for LOD and LOD of Impurity

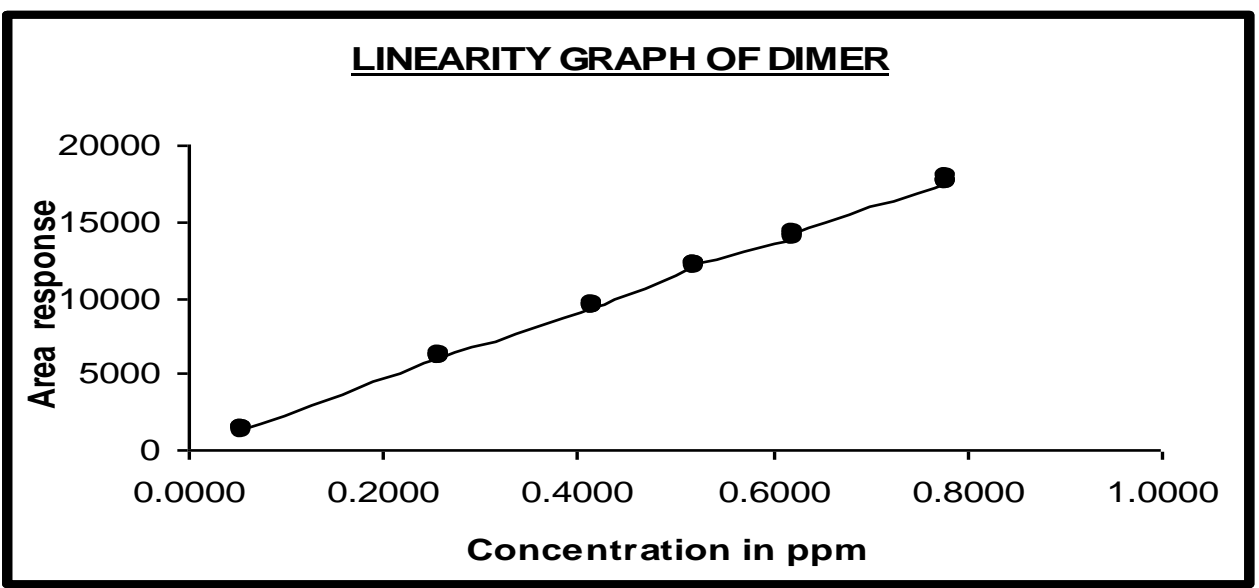

\begin{tabular}{|c|c|}
\hline Intercept(a) & 172.95 \\
\hline Slope(b) & $\mathbf{2 2 4 9 1 . 4 7}$ \\
\hline R2 & 0.9990 \\
\hline R & 0.9995 \\
\hline Standard error & 182.28 \\
\hline Residual sum of squares & $\mathbf{5 3 1 6 1 9 . 2 8}$ \\
\hline
\end{tabular}

Fig.7: Linearity and Range of Impurity

b: Six Replicate of Impurity for Precision Study

\begin{tabular}{|c|c|}
\hline No of Injection & Area of Dimer \\
\hline 1 & 11552 \\
\hline 2 & 11625 \\
\hline 3 & 11784 \\
\hline 4 & 11589 \\
\hline 5 & 11799 \\
\hline 6 & 11550 \\
\hline Mean & $\mathbf{1 1 6 4 9 . 8 3}$ \\
\hline SD & $\mathbf{1 1 3 . 2 3 1 5}$ \\
\hline \%RSD & $\mathbf{0 . 9 7}$ \\
\hline
\end{tabular}

Table 2: Impurity for Accuracy Study 
Impurity Profiling Of Pharmaceutical Drugs By Various Methods

\begin{tabular}{|c|c|c|c|c|c|c|c|c|}
\hline Level & $\begin{array}{c}\text { Solution } \\
\text { No. }\end{array}$ & $\begin{array}{l}\text { Conc. of } \\
\text { DIMER } \\
(\mathrm{ppm})(\mathrm{C})\end{array}$ & $\begin{array}{l}\text { Area counts in } \\
\text { test }+ \text { added } \\
\text { Dimer }\end{array}$ & $\begin{array}{l}\text { Corrected area (After } \\
\text { subtracting the parent } \\
\text { sample area of } \\
\text { Dimer(A) }\end{array}$ & $\begin{array}{l}\text { Recovered } \\
\text { Quantity in } \\
\text { ppm } \\
\text { (B) }\end{array}$ & $\begin{array}{c}\% \\
\text { Recovery }\end{array}$ & $\begin{array}{r}\text { Mean } \\
(n=3)\end{array}$ & \begin{tabular}{|c|}
$\begin{array}{c}\% \text { RSD } \\
(\mathrm{n}=3) \\
\text { Of } \\
\% \text { Recovery }\end{array}$ \\
\end{tabular} \\
\hline \multirow{3}{*}{ LOQ } & Test-1 & \multirow{3}{*}{0.572} & 1443 & 1443 & 0.0644 & 112.59 & \multirow{3}{*}{110.20} & \multirow{3}{*}{3.35} \\
\hline & Test-2 & & 1436 & 1436 & 0.0641 & 112.06 & & \\
\hline & Test-3 & & 1358 & 1358 & 0.0606 & 105.94 & & \\
\hline \multirow{3}{*}{$80 \%$} & Test-1 & \multirow{3}{*}{0.4160} & 9303 & 9303 & 0.4152 & 99.81 & \multirow{3}{*}{99.62} & \multirow{3}{*}{1.87} \\
\hline & Test-2 & & 9102 & 9102 & 0.4063 & 97.67 & & \\
\hline & Test-3 & & 9448 & 9448 & 0.4217 & 101.37 & & \\
\hline \multirow{3}{*}{$100 \%$} & Test-1 & \multirow{3}{*}{0.5200} & 11638 & 11638 & 0.5195 & 99.90 & \multirow{3}{*}{101.40} & \multirow{3}{*}{1.29} \\
\hline & Test-2 & & 11920 & 11920 & 0.5321 & 102.33 & & \\
\hline & Test-3 & & 11881 & 11881 & 0.5303 & 101.98 & & \\
\hline \multirow{3}{*}{$120 \%$} & Test-1 & \multirow{3}{*}{0.6240} & 14444 & 14444 & 0.6447 & 103.32 & \multirow{3}{*}{102.97} & \multirow{3}{*}{0.30} \\
\hline & Test-2 & & 14288 & 14288 & 0.6378 & 102.21 & & \\
\hline & Test-3 & & 14452 & 14452 & 0.6451 & 103.38 & & \\
\hline & & & & & Mean & 103.55 & & \\
\hline & & & & & SD & 4.5960 & & \\
\hline & & & & & RSD & 4.63 & & \\
\hline
\end{tabular}

Table 3: Limit of Detection and Limit of Quantification of Impurity

\begin{tabular}{|c|c|c|c|c|c|c|}
\hline No of Injections & Level & ppm & Area & Regression & y-Regg & $(y-\operatorname{Regg})^{2}$ \\
\hline 1 & \multirow{3}{*}{$10 \%$} & 0.0520 & 1192 & 1223.99 & -31.99 & 1023.38 \\
\hline 2 & & 0.0520 & 1006 & 1223.99 & -217.99 & 47519.76 \\
\hline 3 & & 0.0520 & 994 & 1223.99 & -229.99 & 52895.53 \\
\hline 1 & \multirow{3}{*}{$20 \%$} & 0.1040 & 2464 & 2377.78 & 86.22 & 7434.68 \\
\hline 2 & & 0.1040 & 2565 & 2377.78 & 187.22 & 35053.04 \\
\hline 3 & & 0.1040 & 2355 & 2377.78 & -22.78 & 518.72 \\
\hline 1 & \multirow{3}{*}{$30 \%$} & 0.1560 & 3672 & 3531.56 & 140.44 & 19723.23 \\
\hline 2 & & 0.1560 & 3710 & 3531.56 & 178.44 & 31840.63 \\
\hline 3 & & 0.1560 & 3473 & 3531.56 & -58.56 & 3429.34 \\
\hline 1 & \multirow{3}{*}{$50 \%$} & 0.2600 & 6002 & 5839.13 & 162.87 & 26526.36 \\
\hline 2 & & 0.2600 & 5855 & 5839.13 & 15.87 & 251.83 \\
\hline 3 & & 0.2600 & 5849 & 5839.13 & 9.87 & 97.40 \\
\hline 1 & \multirow{3}{*}{$80 \%$} & 0.4160 & 9207 & 9300.49 & -93.49 & 8739.69 \\
\hline 2 & & 0.4160 & 9178 & 9300.49 & -122.49 & 15002.89 \\
\hline 3 & & 0.4160 & 9291 & 9300.49 & -9.49 & 89.99 \\
\hline 1 & \multirow{3}{*}{$100 \%$} & 0.5200 & 11750 & 11608.06 & 141.94 & 20147.93 \\
\hline 2 & & 0.5200 & 11573 & 11608.06 & -35.06 & 1228.96 \\
\hline 3 & & 0.5200 & 11507 & 11608.06 & -101.06 & 10212.43 \\
\hline
\end{tabular}

Table 4 :Linearity and Range of Impurity

\begin{tabular}{|c|c|c|c|c|c|c|}
\hline No of Injection & Level & ppm & Area & Regression & y-Regg & $(y-R e g g)^{2}$ \\
\hline 1 & \multirow{3}{*}{$\begin{array}{c}\mathrm{LOQ} \\
\%\end{array}$} & 0.0572 & 1294 & 1459.46 & -165.46 & 27376.05 \\
\hline 2 & & 0.0572 & 1400 & 1459.46 & -59.46 & 3535.15 \\
\hline 3 & & 0.0572 & 1359 & 1459.46 & -100.46 & 10091.63 \\
\hline 1 & \multirow{3}{*}{$50 \%$} & 0.2600 & 6055 & 6020.73 & 34.27 & 1174.65 \\
\hline 2 & & 0.2600 & 6184 & 6020.73 & 163.27 & 26658.12 \\
\hline 3 & & 0.2600 & 6145 & 6020.73 & 124.27 & 15443.82 \\
\hline 1 & \multirow{3}{*}{$80 \%$} & 0.4160 & 9395 & 9529.40 & -134.40 & 18062.25 \\
\hline 2 & & 0.4160 & 9557 & 9529.40 & 27.60 & 761.99 \\
\hline 3 & & 0.4160 & 9432 & 9529.40 & -97.40 & 9485.96 \\
\hline 1 & \multirow{3}{*}{$100 \%$} & 0.5200 & 12020 & 11868.51 & 151.49 & 22949.65 \\
\hline 2 & & 0.5200 & 12248 & 11868.51 & 379.49 & 144013.74 \\
\hline 3 & & 0.5200 & 12126 & 11868.51 & 257.49 & 66301.83 \\
\hline 1 & \multirow{3}{*}{$120 \%$} & 0.6240 & 13923 & 14207.62 & -284.62 & 81009.27 \\
\hline 2 & & 0.6240 & 14074 & 14207.62 & -133.62 & 17854.65 \\
\hline 3 & & 0.6240 & 14223 & 14207.62 & 15.38 & 236.51 \\
\hline
\end{tabular}




\section{Conclusion}

Isolating the impurities, purity in given bulk drug can be tested with ease. Assay calculation and factors like bio - availability of the API can be known many effectively using analytical instruments. Beneficial to the society if the drug/ medicine is made available in its purest form so that it acts only on the body part affected and will have minimum side effects. An unknown impurity was identified by HPLC in Dimer was isolated using semi-preparative HPLC for characterization. The structural characterization of this isolated impurity was carried out by using Ion trap LCMS, LC/HR/AM/MS and modern spectroscopic NMR techniques. The combined result of LC/HR/AM/MS and NMR confirmed the structure of unknown impurity 1, 1-bis-(7-chloro-4-hydroxy-3quinolyl\}-ethane. A rapid Acquity H-class liquid chromatographic method developed was

\section{Acknowledgement}

The authors wish to thanks to the professors for supporting this research work. Author thanks to Mukesh Kumar Gupta, Nitin G. Rathod, IPCA laboratories, M.R. Chaudhari, Mumbai for their cooperation \& help.

\section{References}

[1]. M. V. Lokhande, N.G. Rathod, M.K.. Gupta,Structural elucidation, Identification, quantization of process related impurity in Hydralazine Hydrochloride HR/AM- LC MS/MS, NMR and FTIR technique, Journal of Applied Chemistry,6(2),2013, 05-15.

[2]. D. Harvey, Modern Analytical Chemistry, Mc-Graw-Hill higher education publication, 2000, 798-803.

[3]. Guidance for industry: Q3A Impurities in new Drug Substances, 2003.

[4]. ICH Guideline, Validation of Analytical Procedures: Text and Methodology Q2 (R1).

[5]. G. Gauglitz, T. Vo-dinh, Handbook of Spectroscopy, Wiley-VCH Verlag GmbH and co.2003, 538-542.

[6]. J.B. Lambert, E.P. Mazola, Nuclear Magnetic Resonance Spectroscopy, An Introduction to principles, Applications and Experimental methods, Pearson Education Inc. 2004, 341-345.

[7]. J.M. Hollis, Modern Spectroscopy, $4^{\text {th }}$ Edn, Wiley and Sons, Ltd, 2004, 452-458.

[8]. M.W. Dong, Modern HPLC for Practicing Scientists, Dynamics pharmaceutical service LLC, Wiley Interference John Wiley \& sons, 2006, 286-295

[9]. P.K.Braithwaite Smith, Chromatographic methods, Kluwer Academic publishers, 5th Edn. 1999,559-65.

[10]. B. Ardrey ,Liquid Chromatography ,"Mass Spectrometry: An introduction ,2009,10-17

[11]. Guidance for quality risk management Q9, ICH Harmonized Triplicate guideline, 2003.

[12]. M.K.Gupta, M.V.Lokhande , Structure Determination Of Impurity In Memantine Hydrochloride By Analytical Techniques , International Journal of General Medicine and Pharmacy , 3(4), 2014, 75-84.

[13]. M.V. Lokhande, N.G. Rathod, M.K. Gupta, Identification and structural elucidation of process related impurities in duloxetine $\mathrm{HCl}$,International Journal of Chemistry and Pharmaceutical Sciences, 4, 2013, 34-43 .

[14]. A.A.John Chromatographic Analysis of Pharmaceuticals, 2,1997, 135-142.

[15]. L. Yang, Materials Characterization: Introduction to Microscopic and Spectroscopic Methods, Wiley,2009.

IOSR Journal of Applied Chemistry (IOSR-JAC) is UGC approved Journal with S1. No. 4031, Journal no. 44190.

Manohar V.Lokhande. "Impurity Profiling of Pharmaceutical Drugs by Various Methods." IOSR Journal of Applied Chemistry (IOSR-JAC) 10.7 (2017): 27-34. 